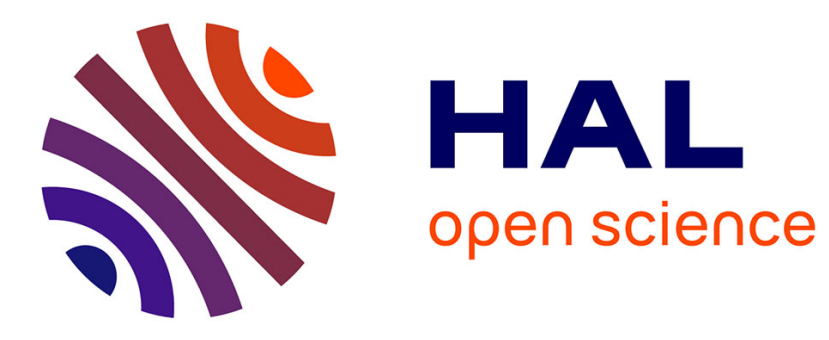

\title{
Joint Morphometry of Fiber Tracts and Gray Matter structures using Double Diffeomorphisms
}

Pietro Gori, Olivier Colliot, Linda Marrakchi-Kacem, Yulia Worbe, Alexandre

Routier, Cyril Poupon, Andreas Hartmann, Nicholas Ayache, Stanley

Durrleman

\section{To cite this version:}

Pietro Gori, Olivier Colliot, Linda Marrakchi-Kacem, Yulia Worbe, Alexandre Routier, et al.. Joint Morphometry of Fiber Tracts and Gray Matter structures using Double Diffeomorphisms. IPMI Information Processing in Medical Imaging, Jun 2015, Isle of Skye, United Kingdom. pp.275-287, 10.1007/978-3-319-19992-4_21. hal-01142628

\section{HAL Id: hal-01142628 \\ https://hal.science/hal-01142628}

Submitted on 15 Apr 2015

HAL is a multi-disciplinary open access archive for the deposit and dissemination of scientific research documents, whether they are published or not. The documents may come from teaching and research institutions in France or abroad, or from public or private research centers.
L'archive ouverte pluridisciplinaire HAL, est destinée au dépôt et à la diffusion de documents scientifiques de niveau recherche, publiés ou non, émanant des établissements d'enseignement et de recherche français ou étrangers, des laboratoires publics ou privés. 


\title{
Joint Morphometry of Fiber Tracts and Gray Matter structures using Double Diffeomorphisms
}

\author{
Pietro Gori $^{1}$, Olivier Colliot ${ }^{1,2}$, Linda Marrakchi-Kacem ${ }^{1,3}$, Yulia Worbe ${ }^{1}$, \\ Alexandre Routier ${ }^{1}$, Cyril Poupon ${ }^{3}$, Andreas Hartmann ${ }^{1}$, Nicholas Ayache ${ }^{4}$ \\ and Stanley Durrleman ${ }^{1}$ \\ 1 Inria Paris-Rocquencourt, Sorbonne Universités, UPMC Univ Paris 06 UMR \\ S1127, Inserm U1127, CNRS UMR 7225, CATI, ICM, F-75013, Paris, France \\ 2 AP-HP, Pitié-Salpêtrière Hospital, Departments of Neurology and Neuroradiology, \\ F-75013, Paris, France \\ 3 Neurospin, CEA, Gif-Sur-Yvette, France \\ 4 Asclepios project-team, Inria Sophia Antipolis, Sophia Antipolis, France
}

\begin{abstract}
This work proposes an atlas construction method to jointly analyse the relative position and shape of fiber tracts and gray matter structures. It is based on a double diffeomorphism which is a composition of two diffeomorphisms. The first diffeomorphism acts only on the white matter keeping fixed the gray matter of the atlas. The resulting white matter, together with the gray matter, are then deformed by the second diffeomorphism. The two diffeomorphisms are related and jointly optimised. In this way, the first diffeomorphisms explain the variability in structural connectivity within the population, namely both changes in the connected areas of the gray matter and in the geometry of the pathway of the tracts. The second diffeomorphisms put into correspondence the homologous anatomical structures across subjects. Fiber bundles are approximated with weighted prototypes using the metric of weighted currents. The atlas, the covariance matrix of deformation parameters and the noise variance of each structure are automatically estimated using a Bayesian approach. This method is applied to patients with Tourette syndrome and controls showing a variability in the structural connectivity of the left cortico-putamen circuit.
\end{abstract}

\section{Introduction}

The brain could be seen as an interlinked multi-object anatomical complex. Both gray and white matter consist of different structures (objects) which can be segmented respectively as 3D surfaces (cortical surface and sub-cortical structures) or as sets of 3D tracts (fiber bundles resulting from tractography algorithms). The shape of these objects can then be analysed in order, for instance, to find morphological characteristics of brain disorders. Most studies examine these objects independently focusing on a single anatomical structure [9-13]. Others propose multi-object analysis considering only a particular kind of mesh, either only sub-cortical structures [5-8] or only fiber-bundles [14-16]. However, an abnormal 
brain development due to a neuropsychiatric disorder can influence not only the shape of individual structures but also their organization. An example is the syndrome of Gilles de la Tourette (GTS) which is thought to be associated with mis-connections of the cortico-striato-pallido-thalamic circuits [3]. These circuits need to be analysed as a whole, studying not only the shape of both white and gray matter components but also their relative position.

A technique to study this problematic is the atlas construction. It permits to estimate an average shape complex of the population under study called template complex and the 3D deformations of the embedding space which warp the template complex to the shape complexes of each subject. Previous works $[2$, $5,8,9]$ have defined these $3 \mathrm{D}$ deformations as a diffeomorphism which prevents shape components to intersect, fold or shear during deformation. This allows the joint analysis of series of objects while guaranteeing preservation of the anatomical organization. However, such a global change of coordinates assumes that the relative position between structures in contact with each other does not change across subjects. This implies that a particular fiber bundle should link the same areas of the cortical surface and basal ganglia across the whole population. This assumption precludes the study of changes in structural connectivity which could be caused by an abnormal brain development as in GTS.

To overcome this problem we propose to replace a single diffeomorphism with a double diffeomorphism which is a composition of two diffeomorphisms. Given a template complex, the idea is to use the first diffeomorphism to deform only the white matter of the template while keeping fixed the gray matter and then to use the second diffeomorphism to deform the resulting white matter together with the gray matter. The first diffeomorphism makes the fiber bundles slide on the fixed cortical surface and basal ganglia. It can be seen as a relative motion with respect to the template gray matter considered as a fixed anatomical reference frame delineating the boundaries of the functional and anatomical territories. The second diffeomorphism puts in correspondence the structures of the template with the homologous ones of the subject. It is a global change of coordinates which brings all the structures in the coordinate system of the subject. The two diffeomorphisms are linked since they both deform the white matter and they are optimised together. This permits to find the composition of diffeomorphisms which allows to separate the variations due to a different structural connectivity (first diffeomorphism) to the ones related to a global shape difference (second diffeomorphism). An illustrative matching toy example in Fig.1 clarifies in which situations double diffeomorphisms are necessary.

In order to deal with the considerable amount of fibers resulting from tractography algorithms, we rely on the approximation scheme introduced in [4]. Fiber bundles are approximated with weighted prototypes represented as "tubes". They are chosen among the fibers and their radius is related to the number of fibers approximated. This new representation is based on the metric of weighted currents [4], an extension of the framework of currents. As usual currents, it does not require point-correspondence between fibers or fiber-correspondence between bundles. Two fibers modelled as weighted currents are considered similar if their 

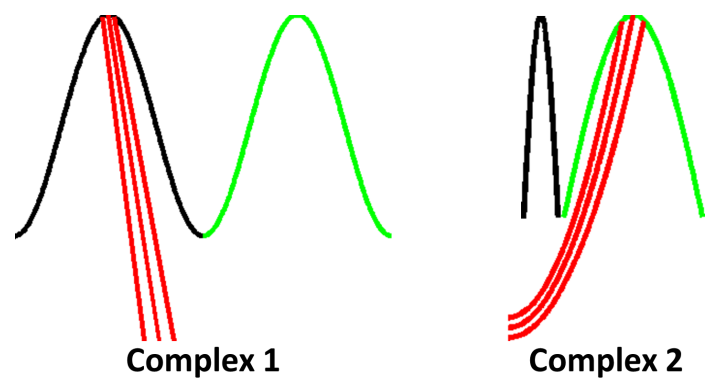

Fig. 1. Two shape complexes composed by a pseudo cortex, divided into a black and green area, and a red pseudo fiber bundle. A single diffeomorphism could not capture the differences in structural connectivity and put in correspondence both structures. A double diffeomorphism would first move the fiber bundle from the left to the right gyrus and then it would change the shape of the gyri, producing an accurate matching.

pathways are alike and their endpoints are close to each other. This metric makes therefore possible to match correctly also the extremities of two fiber bundles and not only their central part as in usual currents. This is fundamental in order to retrieve the connectivity changes at the end of the first diffeomorphism.

The atlas is estimated using a generative statistical model similar to the one in [2] adapted to double diffeomorphisms. The proposed Bayesian model uses similar priors as in [1] which enables to automatically estimate the noise variance of each structure and the covariance matrix of the deformation parameters for both diffeomorphisms. The set of noise variances represent a trade-off between each data-term and the two deformation regularity terms.

In Sect.2, we first introduce how we model the brain structures summarizing the framework of weighted currents and weighted prototypes. We then present the proposed framework of double diffeomorphism and include it into a Bayesian atlas construction method. In Sect.3, we first apply our new scheme to a toy matching example comparing its performance with the one of a single diffeomorphism. Then, we build an atlas with the proposed technique using real data.

\section{Bayesian Double Diffeomorphic Atlas Construction}

\subsection{Object representation}

Gray matter Gray matter objects are modelled as 3D surfaces, where we assume vertex correspondence across subjects. The norm of the difference between two meshes is defined as the sum of squared differences between pair of vertices. White matter Fiber bundles are modelled as weighted currents [4]. Let $X$ and $Y$ be two fibers which can be modelled as polygonal lines of $Q$ and $Z$ segments respectively. We define $\left\{f^{a}, f^{b}\right\}$ for $X$ and $\left\{t^{a}, t^{b}\right\}$ for $Y$ as the vectors containing the coordinates of their extremities. The inner product between these two tracts in the framework of weighted currents is given by: $K_{a}\left(f^{a}, t^{a}\right) K_{b}\left(f^{b}, t^{b}\right)$ 
$\sum_{i=1}^{Q} \sum_{j=1}^{Z} \alpha_{i}^{T} K_{g}\left(x_{i}, y_{j}\right) \beta_{j}$ where $\left\{x_{i}, \alpha_{i}\right\}$ and $\left\{y_{j}, \beta_{j}\right\}$ are the centres and tangent vectors of the segments of $X$ and $Y$ respectively and $K_{a}, K_{b}$ and $K_{g}$ are Gaussian kernels whose bandwidth is fixed by the user. The last one defines the range of interaction between the points of $X$ and $Y$, as in usual currents, while $K_{a}$ and $K_{b}$ set the distances at which extremities of the fibers are considered close. Two fibers are similar if their pathways are alike and if their extremities are close to each other. The space of weighted currents is a vector space, which implies that a fiber bundle $B$ is seen as the sum of its fibers $\left\{F_{i}\right\}: B=\sum_{i} F_{i}$. This makes possible to easily compare two fiber bundles, which do not need to have the same number of fibers, by expanding the inner product $\left\langle\sum_{i} F_{i}, \sum_{j} F_{j}^{\prime}\right\rangle$.

Weighted Prototypes A fiber bundle $B$ is approximated with a set of weighted prototypes $\left\{\tau_{i} M_{i}\right\}$ chosen among the fibers [4]. The prototype $M_{i}$ is modelled as a weighted current and its weight $\tau_{i}$ is linked to the number of fibers approximated. This approximation scheme is controlled by the residual error: $\left\|B-\sum_{i=1}^{K} \tau_{i} M_{i}\right\|_{W^{*}}^{2}$ in the space of weighted currents. It permits to reduce the number of fibers to analyse while preserving connectivity (location of the fiber endpoints on the gray matter) and geometry (pathway of the fibers).

\subsection{Double Diffeomorphic deformation}

Let $N$ be the number of subjects and $M$ the number of objects. All structures of subject $i$ can be seen as a shape complex $\boldsymbol{S}_{i}=\left\{S_{i j}\right\}_{j=1 \ldots M}=\boldsymbol{S}_{i}^{W} \cup \boldsymbol{S}_{i}^{G}$ which is modelled as a double deformation of a common template complex $\boldsymbol{T}=\boldsymbol{T}^{W} \cup \boldsymbol{T}^{G}$ plus a residual noise $\boldsymbol{\epsilon}_{i}=\left\{\boldsymbol{\epsilon}_{i}^{W}, \boldsymbol{\epsilon}_{i}^{G}\right\}$ where $\boldsymbol{T}=\left\{T_{j}\right\}_{j=1 \ldots M}, \boldsymbol{\epsilon}_{i}=\left\{\epsilon_{i j}\right\}_{j=1 \ldots M}$ and the upper indices $W$ and $G$ refer to the White and Gray matter respectively:

$$
\boldsymbol{S}_{i}=\phi_{i}^{A l l}\left(\phi_{i}^{W}\left(\boldsymbol{T}^{W}\right) \cup \boldsymbol{T}^{G}\right)+\boldsymbol{\epsilon}_{i}
$$

The first deformation $\phi_{i}^{W}$ deforms the white matter keeping fixed the gray, thus modeling the changes in the relative position between white and gray matter objects. The second deformation $\phi_{i}^{A l l}$ matches both gray and white matter (the latter already deformed by $\phi_{i}^{W}$ ). Both deformations depend on subject $i$ and they are the last deformation of a flow of diffeomorphisms built by integrating a time-varying vector field $v_{i}(t, x)=v_{i t}(x)\left(t \in[0,1], x \in \boldsymbol{R}^{3}\right)$ (see [5] for details). The two vector fields $v_{i t}^{A l l}(x)$ and $v_{i t}^{W}(x)$ are defined by two different sets of control points $\boldsymbol{c}^{A l l}$ and $\boldsymbol{c}^{W}$ shared among the whole population, and by two sets of 3D vectors, called momenta, $\boldsymbol{\alpha}_{i}^{\text {All }}$ and $\boldsymbol{\alpha}_{i}^{W}$ linked to the control points and specific to each subject $i: v_{i t}^{A l l}(\boldsymbol{x}(t))=\boldsymbol{K}\left(\boldsymbol{x}(t), \boldsymbol{c}^{\text {All }}(t)\right) \boldsymbol{\alpha}_{i}^{A l l}(t)$ and $v_{i t}^{W}(\boldsymbol{x}(t))=\boldsymbol{K}\left(\boldsymbol{x}(t), \boldsymbol{c}^{W}(t)\right) \boldsymbol{\alpha}_{i}^{W}(t)$, where $\boldsymbol{K}(\boldsymbol{x}(t), \boldsymbol{c}(t))$ represents a block matrix of Gaussian kernels with equal fixed width for both deformations. Control points and momenta evolve in time according to the differential equations:

$$
\begin{array}{ll}
\dot{\boldsymbol{c}}_{i}(t)=\boldsymbol{K}\left(\boldsymbol{c}_{i}(t), \boldsymbol{c}_{i}(t)\right) \boldsymbol{\alpha}_{i}(t)=F^{c}\left(\boldsymbol{c}_{i}(t), \boldsymbol{\alpha}_{i}(t)\right) & \boldsymbol{c}_{i}(0)=\boldsymbol{c}(0)=\boldsymbol{c}_{0} \\
\dot{\boldsymbol{\alpha}}_{i}(t)=-\boldsymbol{\alpha}_{i}(t)^{T} \boldsymbol{\alpha}_{i}(t) \nabla_{1} \boldsymbol{K}\left(\boldsymbol{c}_{i}(t), \boldsymbol{c}_{i}(t)\right)=F^{\alpha}\left(\boldsymbol{c}_{i}(t), \boldsymbol{\alpha}_{i}(t)\right) & \boldsymbol{\alpha}_{i}(0)=\boldsymbol{\alpha}_{i 0}
\end{array}
$$


This system of ODEs is valid for both diffeomorphisms: $\boldsymbol{L}_{i}^{A l l / W}(t)=\left\{\boldsymbol{c}_{i}^{A l l / W}(t)\right.$, $\left.\boldsymbol{\alpha}_{i}^{A l l / W}(t)\right\}$ and it can be summarized as $\dot{\boldsymbol{L}}_{i}^{A l l / W}(t)=F\left(\boldsymbol{L}_{i}^{A l l / W}(t)\right)$. The last diffeomorphisms $\phi_{i}^{A l l}(1)$ and $\phi_{i}^{W}(1)$ are completely parametrized by the initial conditions of the systems: $\boldsymbol{L}_{i}^{A l l / W}(0)=\boldsymbol{L}_{i 0}^{A l l / W}=\left\{\boldsymbol{c}_{0}^{A l l / W}, \boldsymbol{\alpha}_{i 0}^{A l l / W}\right\}$. Thus, in order to deform the template complex $\boldsymbol{T}$, we first integrate forward in time $\dot{\boldsymbol{L}}_{i}^{W}(t)$ starting from $\boldsymbol{L}_{i 0}^{W}$ and we use these values to deform only the white objects of the template complex $\left(\boldsymbol{T}^{W}\right)$ integrating forward in time:

$$
\dot{\boldsymbol{T}}_{i}^{W}(t)=\boldsymbol{K}\left(\boldsymbol{T}_{i}^{W}(t), \boldsymbol{c}_{i}^{W}(t)\right) \boldsymbol{\alpha}_{i}^{W}(t) \quad \boldsymbol{T}_{i}^{W}(0)=\boldsymbol{T}_{i 0}^{W}
$$

The deformed white matter template, together with the un-deformed gray matter template $\left(\boldsymbol{T}^{G}\right)$, are then used as starting point for the second deformation All: $\boldsymbol{T}^{A l l}(0)=\boldsymbol{T}^{W}(1) \cup \boldsymbol{T}^{G}(0) . \dot{\boldsymbol{L}}_{i}^{A l l}(t)$ is integrated forward in time starting from $\boldsymbol{L}_{i 0}^{\text {All }}$ and the global template $\boldsymbol{T}^{\text {All }}$ is deformed using a similar equation as Eq.3. Omitting the index $i$ for clarity purpose, the composition is computed as:

$$
\left.\begin{array}{l}
\boldsymbol{L}^{W}(0) \\
\boldsymbol{T}^{W}(0)
\end{array}\right\} \phi^{W}\left\{\begin{array}{l}
\boldsymbol{L}^{W}(1) \\
\boldsymbol{T}^{W}(1)
\end{array} \rightarrow \boldsymbol{T}^{W}(1) \cup \boldsymbol{T}^{G}(0)=\begin{array}{r}
\boldsymbol{L}^{A l l}(0) \\
\boldsymbol{T}^{A l l}(0)
\end{array}\right\} \phi^{A l l}\left\{\begin{array}{l}
\boldsymbol{L}^{A l l}(1) \\
\boldsymbol{T}^{A l l}(1)
\end{array}\right.
$$

\subsection{Optimization procedure}

We show here how to estimate the template complex $\boldsymbol{T}=\boldsymbol{T}^{W} \cup \boldsymbol{T}^{G}$ and the deformation parameters $\boldsymbol{L}_{i 0}^{A l l}=\left\{\boldsymbol{c}_{0}^{A l l}, \boldsymbol{\alpha}_{i 0}^{A l l}\right\}, \boldsymbol{L}_{i 0}^{W}=\left\{\boldsymbol{c}_{0}^{W}, \boldsymbol{\alpha}_{i 0}^{W}\right\}$ which characterize respectively the invariants and the variability of the set of anatomical configurations. This is performed using a Bayesian framework like in $[1$, $2,17]$. Assuming independence between the variables, we model $\boldsymbol{\alpha}_{i 0}^{A l l}$ and $\boldsymbol{\alpha}_{i 0}^{W}$ as multivariate Gaussian variables: $p\left(\boldsymbol{\alpha}_{i 0}^{W} \mid \Gamma_{\alpha}^{W}\right) \sim N\left(0, \Gamma_{\alpha}^{W}\right), p\left(\boldsymbol{\alpha}_{i 0}^{A l l} \mid \Gamma_{\alpha}^{A l l}\right) \sim$ $N\left(0, \Gamma_{\alpha}^{A l l}\right)$ as well as the residual noise $\boldsymbol{\epsilon}_{i}: p\left(\epsilon_{i j}^{G} \mid \sigma_{j}^{2}\right) \sim N\left(0, \sigma_{j}^{2} I d\right)$ and $p\left(\epsilon_{i j}^{W} \mid \sigma_{j}^{2}\right)$ $\sim N\left(0, \sigma_{j}^{2}\left(K_{j}^{W}\right)^{-1}\right) \propto \frac{1}{\left|\sigma_{j}^{2}\right|^{A_{j} / 2}} \exp \left[-\frac{1}{2 \sigma_{j}^{2}}|| \Pi\left(S_{i j}^{W}-\phi_{i}^{A l l}\left(\phi_{i}^{W}\left(T_{j}^{W}\right)\right)\right) \|_{W_{\Lambda_{j}^{*}}^{*}}^{2}\right]$ where $\Lambda_{j}$ refers to the size of the $j$-th grid on which both the shapes and the template of the j-th white matter structure are projected in order to define probability density functions (space of weighted currents is infinite). Moreover we add priors on $\left\{\sigma_{j}^{2}\right\}, \Gamma_{\alpha}^{A l l}$ and $\Gamma_{\alpha}^{W}$ using Inverse Wishart distributions: $\sigma_{j}^{2} \sim \mathcal{W}^{-1}\left(P_{j}, w_{j}\right)$, $\Gamma_{\alpha}^{A l l} \sim \mathcal{W}^{-1}\left(P_{\alpha}^{A l l}, w_{\alpha}^{A l l}\right), \Gamma_{\alpha}^{W} \sim \mathcal{W}^{-1}\left(P_{\alpha}^{W}, w_{\alpha}^{W}\right)$ where the matrices $P_{\alpha}^{W}, P_{\alpha}^{A l l}$ and the scalars $w_{\alpha}^{W}, w_{\alpha}^{A l l},\left\{P_{j}\right\},\left\{w_{j}\right\}$ are hyper-parameters. Both the template complex $\boldsymbol{T}$ and the control points $\left\{\boldsymbol{c}_{0}^{W}\right\}$ and $\left\{\boldsymbol{c}_{0}^{A l l}\right\}$ have a uniform prior distribution. The parameters $\Theta=\left\{\left\{\sigma_{j}^{2}\right\}, \Gamma_{\alpha}^{A l l}, \Gamma_{\alpha}^{W},\left\{\boldsymbol{c}_{0}^{A l l}\right\},\left\{\boldsymbol{c}_{0}^{W}\right\}, \boldsymbol{T}\right\}$ should be estimated considering $\left\{\boldsymbol{\alpha}_{i 0}^{A l l}\right\}$ and $\left\{\boldsymbol{\alpha}_{i 0}^{W}\right\}$ as latent variables and $\left\{\boldsymbol{S}_{i}\right\}$ as observations using, for instance, Monte Carlo sampling procedures (as in [17]). This process would be very time-consuming and we have thus opted for a faster MAP estimation, where $\left\{\boldsymbol{\alpha}_{i 0}^{A l l}\right\}$ and $\left\{\boldsymbol{\alpha}_{i 0}^{W}\right\}$ are considered as parameters $\Theta$. The (minus) 
$\log$ posterior distribution of $\Theta$ given the observations $\left\{\boldsymbol{S}_{i}\right\}$ is equal to:

$$
\begin{aligned}
& \sum_{j=1}^{M} \sum_{i=1}^{N} \frac{1}{2 \sigma_{j}^{2}}\left(\left\|S_{i j}-T_{i j}^{A l l}(1)\right\|^{2}+\frac{P_{j} w_{j}}{N}\right)+\sum_{j=1}^{M} \frac{1}{2}\left(w_{j}+\Lambda_{j} N\right) \log \left(\sigma_{j}^{2}\right)+ \\
& \frac{1}{2} \sum_{i=1}^{N}\left(\boldsymbol{\alpha}_{i 0}^{A l l}\right)^{T}\left(\Gamma_{\alpha}^{A l l}\right)^{-1} \boldsymbol{\alpha}_{i 0}^{A l l}+\frac{\left(w_{\alpha}^{A l l}+N\right)}{2} \log \left(\left|\Gamma_{\alpha}^{A l l}\right|\right)+\frac{w_{\alpha}^{A l l}}{2} \operatorname{tr}\left(\left(\Gamma_{\alpha}^{A l l}\right)^{-1} P_{\alpha}^{A l l}\right)+ \\
& \frac{1}{2} \sum_{i=1}^{N}\left(\boldsymbol{\alpha}_{i 0}^{W}\right)^{T}\left(\Gamma_{\alpha}^{W}\right)^{-1} \boldsymbol{\alpha}_{i 0}^{W}+\frac{\left(w_{\alpha}^{W}+N\right)}{2} \log \left(\left|\Gamma_{\alpha}^{W}\right|\right)+\frac{w_{\alpha}^{W}}{2} \operatorname{tr}\left(\left(\Gamma_{\alpha}^{W}\right)^{-1} P_{\alpha}^{W}\right)
\end{aligned}
$$

$\|\cdot\|$ refers to the norm of weighted currents for white matter objects $\left(\|\cdot\|_{W_{\Lambda j}^{*}}\right)$ and to the $L^{2}$ norm for gray matter objects where $\Lambda_{j}$ is equal to the number of vertices. The framed terms refer respectively to the data-terms and to the regularity of both diffeomorphisms. The other terms are due to the use of priors.

Gradient descent This cost function is minimized using a gradient descent scheme. $\left\{\sigma_{j}^{2}\right\}, \Gamma_{\alpha}^{A l l}, \Gamma_{\alpha}^{W}$ have a closed form solution which is equal to: $\hat{\Gamma}_{\alpha}=$ $\frac{\sum_{i=1}^{N}\left[\left(\boldsymbol{\alpha}_{i 0}\right)\left(\boldsymbol{\alpha}_{i 0}\right)^{T}\right]+w_{\alpha} P_{\alpha}^{T}}{\left(w_{\alpha}+N\right)}$, which is the weighted sum between the sample covariance matrix of the deformation parameters and the prior, and to $\hat{\sigma}_{j}^{2}=$ $\frac{\sum_{i=1}^{N}\left\|S_{i j}-T_{i j}^{A l l}(1)\right\|^{2}+w_{j} P_{j}}{\left(w_{j}+N \Lambda_{j}\right)}$, which is also a weighted sum between the data term of the $j$-th object and the prior. In order to compute the derivatives with respect to $\left\{\boldsymbol{c}_{0}^{A l l}\right\},\left\{\boldsymbol{c}_{0}^{W}\right\}, \boldsymbol{T}^{W}, \boldsymbol{T}^{G},\left\{\boldsymbol{\alpha}_{i 0}^{A l l}\right\}$ and $\left\{\boldsymbol{\alpha}_{i 0}^{W}\right\}$, we rewrite the cost function as:

$$
\begin{aligned}
& E\left[\boldsymbol{T}_{0}^{W}, \boldsymbol{T}_{0}^{G},\left\{\boldsymbol{c}_{0}^{A l l}\right\},\left\{\boldsymbol{\alpha}_{i 0}^{A l l}\right\},\left\{\boldsymbol{c}_{0}^{W}\right\},\left\{\boldsymbol{\alpha}_{i 0}^{W}\right\}\right]=\sum_{i=1}^{N} D_{i}\left[\boldsymbol{T}_{i}^{A l l}(1)\right]+R\left[\boldsymbol{L}_{i 0}^{A l l}\right]+R\left[\boldsymbol{L}_{i 0}^{W}\right] \\
& \text { subject to } \begin{cases}\dot{\boldsymbol{L}}_{i}^{A l l}(t)=F\left[\boldsymbol{L}_{i}^{A l l}(t)\right] & \boldsymbol{L}_{i}^{A l l}(0)=\boldsymbol{L}_{i 0}^{A l l}=\left\{\boldsymbol{c}_{0}^{A l l}, \boldsymbol{\alpha}_{i 0}^{A l l}\right\} \\
\dot{\boldsymbol{L}}_{i}^{W}(t)=F\left[\boldsymbol{L}_{i}^{W}(t)\right] & \boldsymbol{L}_{i}^{W}(0)=\boldsymbol{L}_{i 0}^{W}=\left\{\boldsymbol{c}_{0}^{W}, \boldsymbol{\alpha}_{i 0}^{W}\right\} \\
\dot{\boldsymbol{T}}_{i}^{W}(t)=G\left[\boldsymbol{T}_{i}^{W}(t), \boldsymbol{L}_{i}^{W}(t)\right] & \boldsymbol{T}^{W}(0)=\boldsymbol{T}_{0}^{W} \\
\dot{\boldsymbol{T}}_{i}^{A l l}(t)=G\left[\boldsymbol{T}_{i}^{A l l}(t), \boldsymbol{L}_{i}^{A l l}(t)\right] & \boldsymbol{T}_{i}^{A l l}(0)=\boldsymbol{T}_{i}^{W}(1) \cup \boldsymbol{T}^{G}(0)\end{cases}
\end{aligned}
$$

where $G\left[\boldsymbol{T}_{i}(t), \boldsymbol{L}_{i}(t)\right]=\boldsymbol{K}\left(\boldsymbol{T}_{i}(t), \boldsymbol{c}_{i}(t)\right) \boldsymbol{\alpha}_{i}(t)$ and $D_{i}\left[\boldsymbol{T}_{i}^{A l l}(1)\right], R\left[\boldsymbol{L}_{i 0}^{A l l}\right]$ and $R\left[\boldsymbol{L}_{i 0}^{W}\right]$ are respectively the first, second and third framed terms of Eq.4. Using the calculus of variations as in $[5,18]$ (see Appendix) we obtain:

$$
\begin{cases}\nabla_{\boldsymbol{c}_{0}^{A l}} E=\sum_{i=1}^{N} \xi_{c i}^{A l l}(0)+\nabla_{\boldsymbol{c}_{0}^{A l}} R\left[\boldsymbol{L}_{i 0}^{A l l}\right] & \nabla_{\boldsymbol{c}_{0}^{W}} E=\sum_{i=1}^{N} \xi_{c i}^{W}(0)+\nabla_{\boldsymbol{c}_{0}^{W}} R\left[\boldsymbol{L}_{i 0}^{W}\right] \\ \nabla_{\boldsymbol{\alpha}_{i 0}^{A l l}} E=\xi_{\alpha i}^{A l l}(0)+\nabla_{\boldsymbol{\alpha}_{i 0}^{A l l}} R\left[\boldsymbol{L}_{i 0}^{A l l}\right] & \nabla_{\boldsymbol{\alpha}_{i 0}^{W}} E=\xi_{\alpha i}^{W}(0)+\nabla_{\boldsymbol{\alpha}_{i 0}^{W}} R\left[\boldsymbol{L}_{i 0}^{W}\right] \\ \nabla_{\boldsymbol{T}_{0}^{G}} E=\sum_{i=1}^{N} \theta_{i}^{A l l, G}(0) & \nabla_{\boldsymbol{T}_{0}^{W} E} E \sum_{i=1}^{N} \theta_{i}^{W}(0)\end{cases}
$$


where $\xi_{i}^{A l l}=\left\{\xi_{\alpha i}^{A l l}, \xi_{c i}^{A l l}\right\}, \xi_{i}^{W}=\left\{\xi_{\alpha i}^{W}, \xi_{c i}^{W}\right\}, \theta_{i}^{A l l}=\left\{\theta_{i}^{A l l, G}, \theta_{i}^{A l l, W}\right\},\left\{\theta_{i}^{W}\right\}$ satisfy:

$$
\begin{cases}\dot{\theta}_{i}^{A l l}(t)=-\left(\partial_{\boldsymbol{T}_{i}^{A l l}} G_{i}^{A l l}(t)\right)^{T} \theta_{i}^{A l l}(t) & \theta_{i}^{A l l}(1)=\nabla_{\boldsymbol{T}_{i}^{A l l}(1)} D_{i} \\ \dot{\theta}_{i}^{W}(t)=-\left(\partial_{\boldsymbol{T}_{i}^{W}} G_{i}^{W}(t)\right)^{T} \theta_{i}^{W}(t) & \boldsymbol{\theta}_{\boldsymbol{i}}^{W}(\mathbf{1})=\boldsymbol{\theta}_{\boldsymbol{i}}^{\text {All }}, \boldsymbol{W} \\ (\mathbf{0}) \\ \dot{\xi}_{i}^{A l l}(t)=-\left(\partial_{\boldsymbol{L}_{i}^{A l l}} G_{i}^{A l l}(t)\right)^{T} \theta_{i}^{A l l}(t)+\left(d_{\boldsymbol{L}_{i}^{A l l}} F_{i}^{A l l}(t)\right)^{T} \xi_{i}^{A l l}(t) & \xi_{i}^{A l l}(1)=0 \\ \dot{\xi}_{i}^{W}(t)=-\left(\partial_{\boldsymbol{L}_{i}^{W}} G_{i}^{W}(t)\right)^{T} \theta_{i}^{W}(t)+\left(d_{\boldsymbol{L}_{i}^{W}} F_{i}^{W}(t)\right)^{T} \xi_{i}^{W}(t) & \xi_{i}^{W}(1)=0\end{cases}
$$

where $G_{i}^{A l l}(t)=G\left[\boldsymbol{T}_{i}^{A l l}(t), \boldsymbol{L}_{i}^{A l l}(t)\right], G_{i}^{W}(t)=G\left[\boldsymbol{T}_{i}^{W}(t), \boldsymbol{L}_{i}^{W}(t)\right], F_{i}^{A l l}(t)=F\left[\boldsymbol{L}_{i}^{A l l}(t)\right]$ and $F_{i}^{W}(t)=F\left[\boldsymbol{L}_{i}^{W}(t)\right]$. Once obtained $\boldsymbol{T}_{1}^{\text {All }}$ from current values of control points and momenta, it is used to compute the gradient of the data terms $\nabla_{\boldsymbol{T}_{i}^{\text {All }}(1)} D_{i}$. This information is brought back at time 0 by integrating backward the ODEs of the All auxiliary variables in Eq.7. These results are used to update $\boldsymbol{c}_{0}^{A l l},\left\{\boldsymbol{\alpha}_{i 0}^{A l l}\right\}, \boldsymbol{T}_{0}^{G}$ whereas the values concerning the white matter objects $\left(\theta_{i}^{A l l, W}(0)\right)$ are used as final values of the variable $\theta_{i}^{W}$. This is the key element that connects the two diffeomorphisms. The ODEs of the $W$ auxiliary variables in Eq.7 are then integrated backward and these values are used to update $\boldsymbol{c}_{0}^{W},\left\{\boldsymbol{\alpha}_{i 0}^{W}\right\}, \boldsymbol{T}_{0}^{W}$. ODEs are integrated with the Euler's method using 10 steps. Here, the flow of information goes from All to $W$, contrary to Sect.2.2, and it can be summed up as:

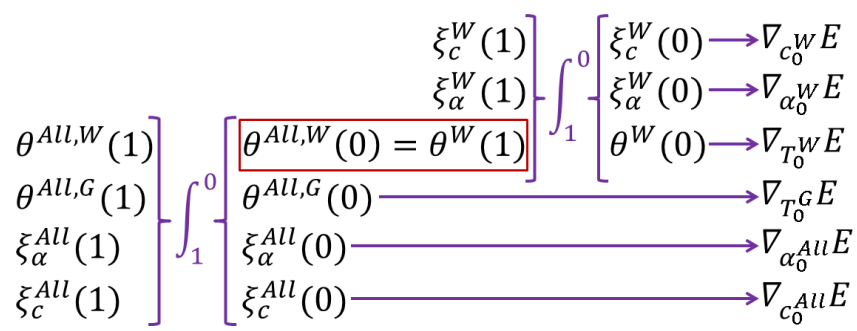

\section{$3 \quad$ Experiments}

Fig.2 shows a comparison between a single and a double diffeomorphic matching applied to a toy example. The structural connectivity is different since the fiber bundles end in different folds of the cortex. Using a single diffeomorphism the cortical surface changes its folding since a peak becomes a valley and vice-versa (see the four coloured $X$ ), and at the same time the fiber bundles are not well matched. Instead, using a double diffeomorphism we can first see the relative motion of the fiber bundles with respect to the fixed cortex and basal ganglia which highlights the differences in structural connectivity. Then, all structures are well matched via the second global diffeomorphism. In this case the cortex is simply shifted without modifying its folding structure, which is more sensible 
since the two cortical surfaces have the same number of folds.

We have also built an atlas with 2 controls and 3 patients subject to GTS using left cortex, putamen and the fiber bundle connecting them. The cortex is segmented using FreeSurfer and the putamen with FSL, in both cases there is a vertex-correspondence between subjects. The fiber bundle comes from a deterministic tractography [3]. The templates of cortex and putamen have been initialised as the average of the vertices. For the fiber bundle template, we have first gathered the fibers of all subjects in a single bundle which has then been approximated as a set of weighted prototypes [4]. Both Fig.3 and Fig.4 show the first mode of PCA based on the estimated covariance matrix of the deformation parameters of the first diffeomorphism $W$ applied to the fiber bundle template keeping fixed the templates of cortex and putamen. Given the important structural changes that are likely to occur in GTS patients [3], we may assume that controls and patients create separate clusters, so that the first mode of PCA on the pooled data mostly highlights the effects of the pathology on the anatomy and structural connectivity. Fig.3 highlights the displacement of the points from the estimated template along the first mode at plus and minus three times the standard deviation $\sigma$. This shows the variability of the geometry of the fibers. In Fig.4 we analyse the changes of the probability density of the endpoints of the fibers on the fixed gray matter templates. This permits to point out the main differences in the connected areas. Changes in connections concern mainly the frontal and parietal lobe of the cortex and the dorsal part of the putamen.

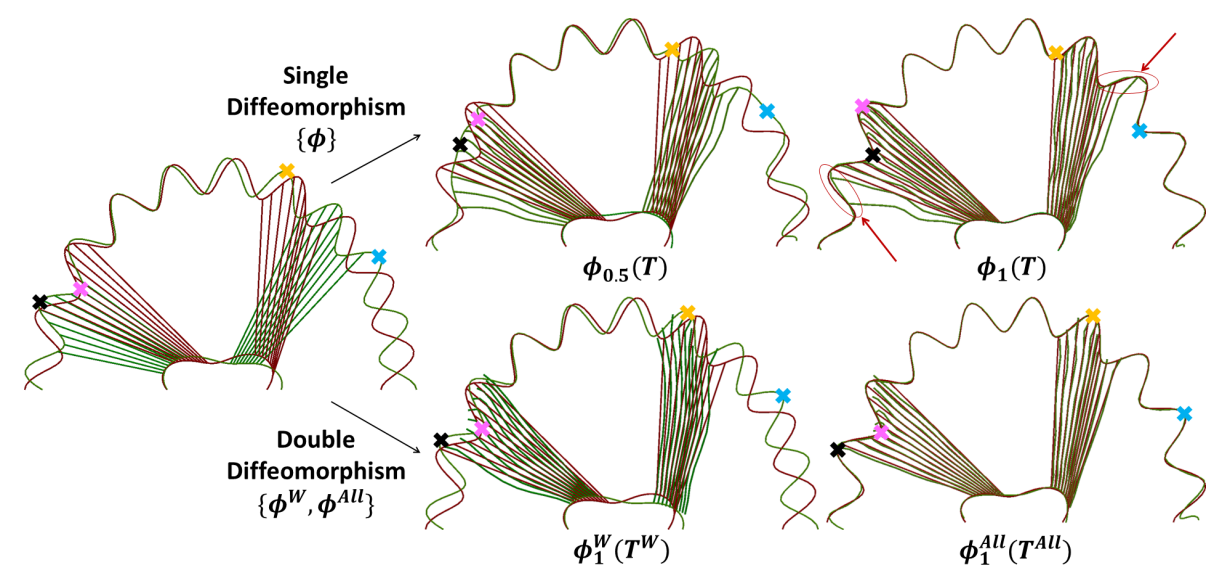

Fig. 2. Matching between the green structures towards the red ones. In the first row it is used one diffeomorphism $(\phi)$ whereas in the second row it is shown the proposed double diffeomorphic scheme $\phi^{W} \cup \phi^{A l l}$. These four structures represent the cortical surface, the basal ganglia and two fiber bundles connecting them. The first two are modelled as varifolds (see [5]) and the last two as weighted currents. We have used the same modeling and deformation parameters for the two settings. The four coloured $\boldsymbol{X}$ represent some peaks and valleys that are inverted in the first row and conserved in the second one. The red arrows highlight the matching errors using one diffeomorphism. 

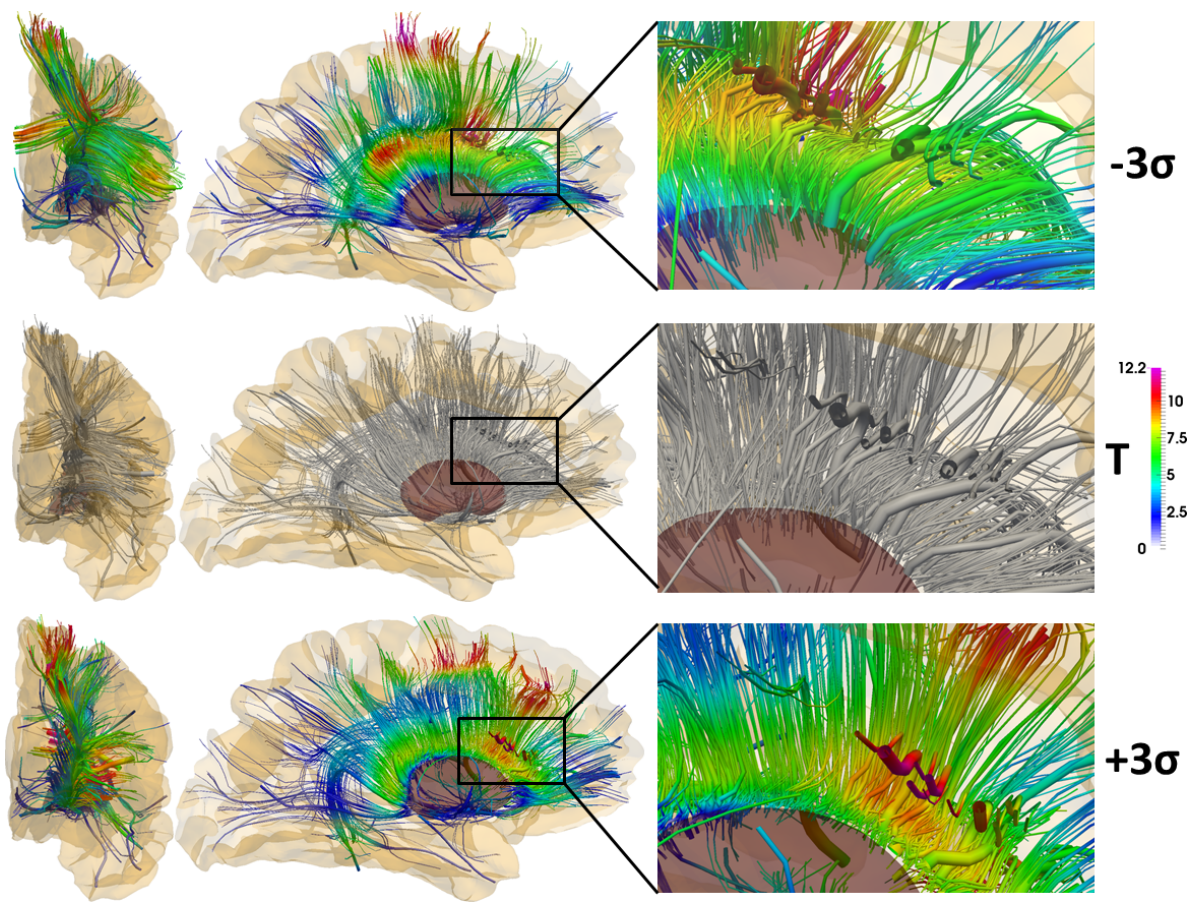

Fig. 3. Displacement of the fiber bundle template deformed with the first mode of PCA based on the covariance matrix of the deformation parameters of the first diffeomorphism at $\pm 3 \sigma$, keeping fixed the gray matter templates. Colors refer to the magnitude of the displacement from the template, shown in gray in the middle row.

\section{Conclusions and Discussion}

We have presented a method to study the variability of the structural connectivity within a population using a multi-object atlas procedure based on a double diffeomorphism. It permits to test the hypothesis that fiber bundles can link different areas of the gray matter structures across the population, which was not possible using a single diffeomorphism. This scheme permits also to study the shape of white and gray matter structures typical of the population. A question that naturally arises using the proposed method is about the uniqueness of the decomposition into two diffeomorphisms. Since gray matter objects are deformed uniquely by the second deformation, there might be an ambiguity concerning the regions which contain only white matter structures. In order to obtain a unique decomposition, we have chosen a scale of deformation so that white matter objects are deformed by the second diffeomorphism in a correlated way with respect to at least one gray matter structure. Furthermore, our algorithm is limited for now to cases in which we can assume that there is a point-correspondence among 

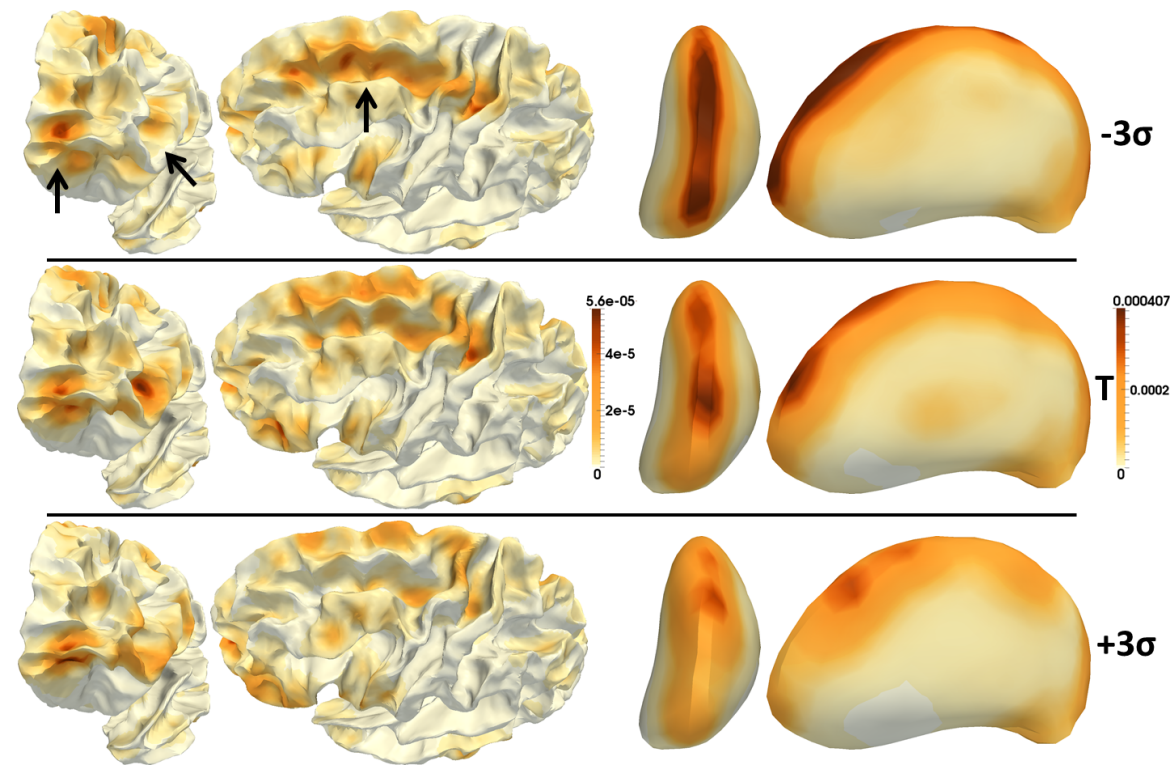

Fig. 4. Fiber bundle template deformed with the first mode of PCA based on the covariance matrix of the deformation parameters of the first diffeomorphism at $\pm 3 \sigma$, keeping fixed the gray matter templates. Colors refer to the probability densities of the endpoints of the fibers onto the gray matter templates.

the cortical surfaces in the population. In future works, we will try to adapt our technique for considering also subjects showing different cortical gyrifications. We have applied this technique to a population of 3 patients with GTS and 2 controls using left cortex, putamen and the fiber bundle connecting them. We have shown that the main variation in structural connectivity within the population is in the frontal and parietal lobe of the cortex and in the dorsal part of the putamen. This could indicate the principal variability between patients and controls. Future works will extend this technique to a larger population considering all the cortico-striato-pallido-thalamic circuits. This will permit to verify our preliminary results about atypical structural connections in the cortico-putamen circuit which likely result from abnormal brain development due to GTS [3].

Acknowledgements The research leading to these results has received funding from the program "Investissements d'avenir" ANR-10-IAIHU-06.

\section{Appendix}

We compute here the gradient of the criterion for atlas construction. A variation in the white system $\delta \boldsymbol{L}_{i 0}^{W}$ produces a variation in the path of control points and momenta $\delta \boldsymbol{L}_{i}^{W}(t)$ and consequently $\delta \boldsymbol{T}_{i}^{W}(t)$. In parallel, $\delta \boldsymbol{L}_{i 0}^{\text {All }}$ produces a variation in $\delta \boldsymbol{L}_{i}^{A l l}(t)$ which, together with $\delta \boldsymbol{T}_{i 1}^{W}$, induces a variation in the global 
template $\delta \boldsymbol{T}_{i}^{A l l}(t)$ and then in the criterion $\delta E$ :

$$
\begin{array}{ll}
\delta E=\sum_{i=1}^{N} \nabla_{\boldsymbol{T}_{i 1}^{A l l}}\left(D_{i}\left[\boldsymbol{T}_{i 1}^{A l l}\right]\right)^{T} \delta \boldsymbol{T}_{i 1}^{A l l}+\nabla_{\boldsymbol{L}_{i 0}^{A l l}}\left(R\left[\boldsymbol{L}_{i 0}^{A l l}\right]\right)^{T} \delta \boldsymbol{L}_{i 0}^{A l l} & +\nabla_{\boldsymbol{L}_{i 0}^{W}}\left(R\left[\boldsymbol{L}_{i 0}^{W}\right]\right)^{T} \delta \boldsymbol{L}_{i 0}^{W} \\
\delta \dot{\boldsymbol{L}}_{i}^{A l l}(t)=\left(d_{\boldsymbol{L}_{i t}^{A l l}} F_{i}^{A l l}(t)\right)^{T} \delta \boldsymbol{L}_{i t}^{A l l} & \delta \boldsymbol{L}_{i}^{A l l}(0)=\delta \boldsymbol{L}_{i 0}^{A l l} \\
\delta \dot{\boldsymbol{L}}_{i}^{W}(t)=\left(d_{\boldsymbol{L}_{i t}^{W}} F_{i}^{W}(t)\right)^{T} \delta \boldsymbol{L}_{i t}^{W} & \delta \boldsymbol{L}_{i}^{W}(0)=\delta \boldsymbol{L}_{i 0}^{W} \\
\delta \dot{\boldsymbol{T}}_{i}^{W}(t)=\left(\partial_{\boldsymbol{T}_{i t}^{W}} G_{i}^{W}(t)\right)^{T} \delta \boldsymbol{T}_{i t}^{W}+\left(\partial_{\boldsymbol{L}_{i t}^{W}} G_{i}^{W}(t)\right)^{T} \delta \boldsymbol{L}_{i t}^{W} & \delta \boldsymbol{T}^{W}(0)=\delta \boldsymbol{T}_{0}^{W} \\
\left.\delta \dot{\boldsymbol{T}}_{i}^{A l l}(t)=\left(\partial_{\boldsymbol{T}_{i t}^{A l l}} G_{i}^{A l l}(t)\right)^{T} \delta \boldsymbol{T}_{i t}^{A l l}+\left(\partial_{\boldsymbol{L}_{i t}^{A l}} G_{i}^{A l l}(t)\right]\right)^{T} \delta \boldsymbol{L}_{i t}^{A l l} & \delta \boldsymbol{T}_{i}^{A l l}(0)=\delta \boldsymbol{T}_{i 1}^{W} \cup \delta \boldsymbol{T}_{0}^{G}
\end{array}
$$

As in [5] we denote: $R_{s t}=\exp \left(\int_{s}^{t} d_{\boldsymbol{L}_{u}} F(u) d u\right)$ and $V_{s t}=\exp \left(\int_{s}^{t} \partial_{\boldsymbol{T}_{u}} G(u) d u\right)$ which are valid for both frameworks $W$ and $A l l$ and where we have omitted the index $i$ for clarity purpose. The two first ODEs are linear whereas the last two are linear with source term: $\delta \boldsymbol{L}(t)=R_{0 t} \delta \boldsymbol{L}_{0}, \delta \boldsymbol{T}(t)=\int_{0}^{t} V_{u t} \partial_{\boldsymbol{L}_{u}} G(u) \delta \boldsymbol{L}(u) d u+$ $V_{0 t} \delta \boldsymbol{T}_{0}$. Calling $\boldsymbol{Y}^{W}$ and $\boldsymbol{Y}^{G}$ the white and gray matter objects of $\boldsymbol{T}^{A l l}$ :

$$
\begin{gathered}
\delta \boldsymbol{Y}^{W}(t)=\left(\int_{0}^{t} V_{u t}^{A l l} \partial_{\boldsymbol{L}^{A l l}} G^{A l l}(u) R_{0 u}^{A l l} d u\right) \delta \boldsymbol{L}_{0}^{A l l}+V_{0 t}^{A l l} \delta \boldsymbol{T}_{0}^{W}+ \\
V_{0 t}^{A l l} \int_{0}^{1} \partial_{\boldsymbol{T}^{W}} G^{W}(s) \delta \boldsymbol{T}^{W}(s) d s+V_{0 t}^{A l l} \int_{0}^{1} \partial_{\boldsymbol{L}^{W}} G^{W}(s) \delta \boldsymbol{L}^{W}(s) d s
\end{gathered}
$$

Using the Fubini's theorem, the $3^{\text {rd }}$ term is equal to: $\left(V_{0 t}^{A l l} \int_{0}^{1} V_{u 1}^{W} \partial_{\boldsymbol{L}^{W}} G^{W}(u) R_{0 u}^{W} d u\right)$ $\delta \boldsymbol{L}_{0}^{W}-\left(V_{0 t}^{A l l} \int_{0}^{1} \partial_{\boldsymbol{L}^{W}} G^{W}(u) R_{0 u}^{W} d u\right) \delta \boldsymbol{L}_{0}^{W}+\left(V_{0 t}^{A l l} V_{01}^{W}\right) \delta \boldsymbol{T}_{0}^{W}-V_{0 t}^{A l l} \delta \boldsymbol{T}_{0}^{W}$. The $4^{\text {th }}$ term becomes: $\left(V_{0 t}^{A l l} \int_{0}^{1} \partial_{\boldsymbol{L}^{W}} G^{W}(s) R_{0 s}^{W} d s\right) \delta \boldsymbol{L}_{0}^{W}$. Plugging them into $\delta E$ :

$$
\begin{aligned}
& \nabla_{\boldsymbol{L}_{0}^{A l l}} E=\left(\int_{0}^{1}\left(R_{0 u}^{A l l}\right)^{T}\left(\partial_{\boldsymbol{L}^{A l l}} G^{A l l}(u)\right)^{T}\left(V_{u 1}^{A l l}\right)^{T} d u\right) \nabla_{\boldsymbol{T}_{1}^{A l l}} D+\nabla_{\boldsymbol{L}_{0}^{A l l}} R^{A l l} \\
& \nabla_{\boldsymbol{L}_{0}^{W}} E=\left(\int_{0}^{1}\left(R_{0 u}^{W}\right)^{T}\left(\partial_{\boldsymbol{L}^{W}} G^{W}(u)\right)^{T}\left(V_{u 1}^{W}\right)^{T} d u\right)\left(V_{01}^{A l l}\right)^{T} \nabla_{\boldsymbol{Y}_{1}^{W}} D+\nabla_{\boldsymbol{L}_{0}^{W}} R^{W} \\
& \nabla_{\boldsymbol{T}_{0}^{W}} E=\left(V_{01}^{W}\right)^{T}\left(V_{01}^{A l l}\right)^{T} \nabla_{\boldsymbol{Y}_{1}^{W}} D \\
& \nabla_{\boldsymbol{T}_{0}^{G}} E=\left(V_{01}^{A l l}\right)^{T} \nabla_{\boldsymbol{Y}_{1}^{G}} D
\end{aligned}
$$

Calling $\theta^{A l l, G}(t)=\left(V_{t 1}^{A l l}\right)^{T} \nabla_{Y_{1}^{G}} D, \theta_{t}^{A l l, W}=\left(V_{t 1}^{A l l}\right)^{T} \nabla_{Y_{1}^{W}} D, \theta^{A l l}(t)=\left\{\theta^{A l l, G}(t)\right.$, $\left.\theta^{A l l, W}(t)\right\}, \theta^{W}(t)=\left(V_{t 1}^{W}\right)^{T} \theta_{0}^{A l l, W}, \xi^{A l l}(t)=\int_{t}^{1}\left(R_{t u}^{A l l}\right)^{T}\left(\partial_{L^{A l l}} G^{A l l}(u)\right)^{T} \theta^{A l l}(u) d u$ and $\xi^{W}(t)=\int_{t}^{1}\left(R_{t u}^{W}\right)^{T}\left(\partial_{\boldsymbol{L}^{W}} G^{W}(u)\right)^{T} \theta^{W}(u) d u$ we obtain the results in Eq.6.

\section{References}

1. Allassonnière S, Amit Y, Trouvé A: Toward a Coherent Statistical Framework for Dense Deformable Template Estimation. JR Statist Soc B, 69(1), 3-29 (2007) 
2. Gori P, Colliot O, Worbe Y, Marrakchi-Kacem L, Lecomte S, Poupon C, Hartmann A, Ayache, N, Durrleman, S: Bayesian Atlas Estimation for the Variability Analysis of Shape Complexes. MICCAI, 16(1), 267-74 (2013)

3. Worbe Y, Marrakchi-Kacem L, Lecomte S, Valabregue R, Poupon F, Guevara P, Tucholka A, Mangin JF, Vidailhet M, Lehericy S, Hartmann A, Poupon C: Altered structural connectivity of cortico-striato-pallido-thalamic networks in Gilles de la Tourette syndrome. Brain. DOI: 10.1093/brain/awu311 (2014)

4. Gori P, Colliot O, Marrakchi-Kacem L, Worbe Y, De Vico Fallani F, Chavez M, Lecomte S, Poupon C, Hartmann A, Ayache N, Durrleman S: A prototype representation to approximate white matter bundles with weighted currents. MICCAI, 17(3), 289-96 (2014)

5. Durrleman S, Prastawa M, Charon N, Korenberg JR, Joshi S, Gerig G, Trouvé A: Morphometry of anatomical shape complexes with dense deformations and sparse parameters. NeuroImage. DOI: 10.1016/j.neuroimage.2014.06.043 (2014)

6. Chen T, Rangarajan A, Eisenschenk SJ, Vemuri BC: Construction of a neuroanatomical shape complex atlas from 3D MRI brain structures. NeuroImage, 60(3), 1778-87 (2012)

7. Gorczowski K, Styner M, Jeong JY, Marron JS, Piven J, Hazlett HC, Pizer SM, Gerig G: Multi-object analysis of volume, pose, and shape using statistical discrimination. IEEE Trans Pattern Anal Mach Intell, 32(4), 652-61 (2010)

8. Qiu A, Miller MI.: Multi-structure network shape analysis via normal surface momentum maps. NeuroImage, 42(4), 1430-8 (2008)

9. Ma J, Miller MI, Younes L: A Bayesian Generative Model for Surface Template Estimation. Int J Biomed Imaging. doi:10.1155/2010/974957 (2010)

10. Davies RH, Twining CJ, Cootes TF, Taylor CJ: Building 3-D Statistical Shape Models by Direct Optimization. IEEE Trans Med Imag, 29(4), 961-81 (2010)

11. Hufnagel H, Pennec X,Ehrhardt J, Ayache N, Handels H: Computation of a Probabilistic Statistical Shape Model in a Maximum-a-posteriori Framework. Methods Inf Med, 48(4), 314-19 (2009)

12. Niethammer M, Reuter M, Wolter FE, Bouix S, Peinecke N, Koo MS, Shenton ME: Global Medical Shape Analysis Using the Laplace-Beltrami Spectrum. MICCAI, 10(1), 850-57 (2007)

13. Joshi SH, Cabeen RP, Joshi AA, Sun B, Dinov I, Narr KL, Toga AW, Woods RP: Diffeomorphic sulcal shape analysis on the cortex. IEEE Trans Med Imaging, 31(6), 1195-212 (2012)

14. Durrleman S, Fillard P, Pennec X, Trouvé A, Ayache N: Registration, Atlas Estimation and Variability Analysis of White Matter Fiber Bundles Modeled as Currents. Neuroimage, 55(3), 1073-90 (2011)

15. O'Donnell LJ, Westin CF, Golby AJ.: Tract-based morphometry for white matter group analysis. Neuroimage, 45(3), 832-44 (2009)

16. Wassermann D, Rathi Y, Bouix S, Kubicki M, Kikinis R, Shenton M, Westin $\mathrm{CF}$ : White matter bundle registration and population analysis based on Gaussian processes. IPMI, 22, 320-32 (2011)

17. Zhang M, Singh N, Fletcher PT: Bayesian estimation of regularization and atlas building in diffeomorphic image registration. IPMI, 23, 37-48 (2013)

18. Marsland S, McLachlan R: A Hamiltonian particle method for diffeomorphic image registration. IPMI, 20, 396-407 (2007) 